\title{
Validación de un módulo de estrategias constructivistas para mejorar la comprensión lectora en inglés - EBA
}

\author{
Validation of a module of constructive strategies to improve reading \\ comprehension in english - EBA
}

\author{
María Julia Alvarado Flores ${ }^{1}$ \\ Liceo Naval de la Marina de Guerra del Perú
}

\author{
Alberto Quintana Peña ${ }^{2}$ \\ Universidad Nacional Mayor de San Marcos
}

Recibido: 26 - $11-18$

Aceptado: $27-12-18$

\begin{abstract}
Resumen
Esta investigación tiene como propósito demostrar la eficacia del empleo de la estrategia constructivista para mejorar la comprensión lectora en el idioma inglés en los estudiantes de Educación Básica Alternativa (EBA) del Ministerio de Educación del Perú. La aplicación de dicha estrategia permite al estudiante involucrarse con el texto, ya que recoge sus experiencias previas como lector, así como de su vida cotidiana; impulsándolo a armar nuevos conceptos, facilitando de esta manera comprender los textos que lee. Se aplica la Prueba de Entrada y Salida "A busy day in the office", que ha logrado los principios de validez y confiabilidad, antes de iniciar la experiencia. Se seleccionó una muestra de 32 estudiantes de primer año del CEBA "Bartolomé Herrera", 16 de la sección "A", grupo de control y 16 de la sección "B" del grupo experimental. Las edades de los estudiantes fluctúan entre 16 a 29 años; de acuerdo con los Objetivos Dakar (2012) el mayor reto de la educación se encuentra en el acceso y finalización de los grupos desfavorecidos o marginados. Este trabajo resume la solución a una problemática: que los alumnos comprendan cuando lean, incrementen su capacidad de retención, deducción-inducción, opinión y recreación (Solé, 2004). La aplicación de las estrategias antes, durante y después de la lectura permite a los estudiantes responder satisfactoriamente a las preguntas literales, inferenciales y crítico-valorativas. Además, el grupo experimental demuestra más perseverancia al trabajar en clase cuando los temas están vinculados a sus necesidades e intereses. Es un instrumento significativo, que recoge los planteamientos de una metodología Constructivista, mediante la cual el lector interactúa con el texto, armando mentalmente ideas e interpretándolas (Pinzás, 2012). En el anexo se considera una ficha de lectura, la imagen y preguntas en cada uno de los niveles de comprensión lectora; así como otras actividades que hicieron posible que los estudiantes comprendan y trabajen los textos.
\end{abstract}

Palabras clave: Educación; comprensión lectora; estrategias constructivistas; inglés y estudiantes.

1 Docente en Liceo Naval del Callao. Autor para correspondencia. E-mail: majualvarado@hotmail.com 2 Decano Facultad de Psicología de la Universidad Nacional Mayor de San Marcos. E-mail: aquintanap@unmsm.edu.pe

(C) Los autores. Este artículo es publicado por la Revista de Investigación en Psicología de la Facultad de Psicología, Universidad Nacional Mayor de San Marcos. Este es un artículo de acceso abierto, distribuido bajo los términos de la licencia Creative Commons Atribucion - No Comercia_Compartir Igual 4.0 Internacional. (http://creativecommons.org/licenses/by-nc-sa/4.0/) que permite el uso no comercial, distribución y reproducción en cualquier medio, siempre que la obra original sea debidamente citada. 


\begin{abstract}
The purpose of this research is to demonstrate the effectiveness of the use of the constructivist strategy to improve reading comprehension in the English language in students of Alternative Basic Education (EBA) of the Ministry of Education of Peru. The application of this strategy allows the student to get involved with the text, since it gathers his previous experiences as a reader, as well as his daily life; pushing it to put together new concepts, facilitating in this way to understand the texts it reads. The "A busy day in the office" Entry and Exit Test is applied, which has achieved the principles of validity and reliability, before starting the experience. A sample of 32 first-year students of the CEBA "Bartolomé Herrera", 16 of the "A" section, control group and 16 of the "B" section of the experimental group was selected. The ages of the students range from 16 to 29 years; According to the Dakar Objectives (2012), the greatest challenge of education lies in the access and completion of disadvantaged or marginalized groups. This work summarizes the solution to a problem: that students understand when they read, increase their capacity for retention, deduction-induction, opinion and recreation (Solé, 2004). The application of the strategies before, during and after the reading allows the students to respond satisfactorily to the literal, inferential and critical-evaluative questions. In addition, the experimental group demonstrates more perseverance when working in class when the topics are linked to their needs and interests. It is a significant instrument, which includes the approaches of a Constructivist methodology, through which the reader interacts with the text, mentally arming ideas and interpreting those (Pinzás, 2012). In the annex a reading card is considered, the image and questions in each one of the levels of reading comprehension; as well as other activities that made it possible for students to understand and work on the texts.
\end{abstract}

Keywords: Education; reading comprehension; constructivist strategies; English and students.

\title{
INTRODUCCIÓN
}

El Constructivismo es una teoría epistemológica, es decir, una explicación acerca de cómo se construye el conocimiento; no es una teoría psicológica ni teoría educativa (Frisancho, 2016). Sin embargo, ayuda a entender qué es lo que sucede en el interior del sujeto cuando incorpora nuevos conocimientos. La educación como práctica social busca formar individuos que puedan desarrollarse y adaptarse a la sociedad (Delval, 2000). El docente facilita la construcción del aprendizaje, es el arquitecto que tiene el reto permanente de recrear las diversas estrategias para que los estudiantes construyan los conocimientos. Conocer es construir conocimientos. (Ganem y Ragasol, 2016).

La lectura es un proceso dinámico de construcción de significados (Gonzáles, M. y otros, 2016), implica el despliegue de un conjunto de habilidades que permiten al lector relacionar e integrar información con la finalidad de comprender el texto en su totalidad, así como reflexionar sobre el texto leído y emitir un juicio crítico sobre algunos de sus elementos. Este proceso ocurre en un contexto social, en el cual los textos son mediadores y, a la vez, producto de la práctica letrada de una comunidad (Solé, 2011).

Además, la lectura es un proceso estratégico e interactivo; estratégico porque el lector decide rutas, caminos, atajos y procedimientos para entender el sentido del texto de acuerdo con el propósito de la lectura (Akbari, 2013). Es interactivo porque 
continuamente el lector elabora hipótesis y deducciones que va contrastando con la información del texto y de su saber previo sobre el mundo, y a su vez utiliza la información como insumo para elaborar nuevas hipótesis (MINEDU, 2015).

La lectura promueve la formación de una persona competitiva en los diversos campos del conocimiento; creativa, capaz de formar su opinión sobre un tema; y con potencialidad de usar la palabra de manera oral o escrita. El desarrollo de destrezas comunicativas promoverá en consecuencia una comunidad más reflexiva y analítica (Idrogo y Huamán, 2018).

La comprensión lectora tiene una vital importancia en el proceso de enseñanzaaprendizaje del idioma inglés (Crystal, 2003) permite que los estudiantes entiendan los textos, aumenten su vocabulario, refuercen las estructuras gramaticales; si lee en voz alta ayudará a la discriminación auditiva de las palabras, pronunciará con corrección y le permitirá mejorar su redacción inglesa. Su relevancia radica en que los estudiantes aprehendan la lengua anglosajona a través de los textos que leen.

El Consejo Británico declara que el idioma inglés es la herramienta que permite la comunicación con personas de diferentes países ya que se enseña como segunda lengua a nivel mundial. Según los datos de la Biblioteca del Congreso de los Estados Unidos un tercio de los libros del mundo son publicados en dicho idioma. Su popularidad y auge es considerable (Crystal, 2003), es más utilizada por personas que lo hablan como segunda lengua, que como lengua materna. En el Perú el ex Ministro de Cultura Luis Peirano (2010) afirmó que saber inglés en esta época es fundamental para lograr un óptimo desarrollo académico y profesional. En un mundo competitivo en el que vivimos, es básico dominar este idioma, para tener acceso a todas las manifestaciones culturales, artísticas, deportivas, científicas, etc. que se producen en todos los niveles del conocimiento. La mayor cantidad y profundidad de información se elabora en inglés (Ullari, 2017).

Los estudiantes de la EBA deben recibir una formación académica de acuerdo a las exigencias de esta época (Mendes, 2014). En una sociedad moderna el sistema escolar educa y contribuye a la democratización de la información y tratará de garantizar a toda la ciudanía una formación básica que responda a las necesidades laborales y realización personal (Aguilar, 2015).

Brindar estrategias de comprensión lectora en inglés es desarrollar las posibilidades de entender textos fuera del ámbito escolar (Sanz, 2009). Por ese motivo, en las aulas de EBA proporciona la calidad que el curso lo requiere, más aun, considerando que muchos de nuestros estudiantes ya se desempeñan laboralmente. La educación debe dar oportunidad al desarrollo pleno de la personalidad humana (UNESCO, 1948).

Las clases de inglés que se imparten promueven motivaciones para pensar y reflexionar en su quehacer, para forjar en el estudiante el constructor de sus 
conocimientos en base a las experiencias que trae (Pinzás, 2012). El 80\% de los estudiantes de EBA son provincianos, alumnas que salieron embarazadas en EBR (Educación Básica Regular), niños y adolescentes que dejaron las aulas para dedicarse a trabajar y ayudar a sus padres, o, en algunos casos se volvieron pandilleros y se retiraron de las aulas por muchos años (MINEDU, 2009). La mayoría de estos estudiantes reingresará al colegio, pero en la modalidad EBA. Teniendo en cuenta la Declaración Mundial sobre Educación para Todos (1994), el proceso educativo debe adaptarse a los cambios; innovar, mantenernos a la vanguardia y exigencias que la sociedad lo requiera. Los alumnos interrumpieron sus estudios durante meses, años, incluso décadas y no se ubican con facilidad en su nuevo contexto, ya que traen experiencias personales, sociales, culturales y educativas diferentes (Ricoy y Alvarez, 2016). El empleo de estrategias Constructivistas permite que el estudiante no se sienta foráneo de los nuevos términos que va a leer, por el contrario, los vinculará con su lengua materna; su valiosa experiencia que tiene en su día a día como madre o padre de familia, trabajador; su mundo laboral (vendedores, mozos, mecánicos, canillitas, empleados, empleadores, etc.) constituyen un gran aporte al nuevo reto de comprender textos en inglés (Briones, 2004).

Enseñar comprensión lectora en inglés en EBA significa tener en cuenta un trabajo en equipo, para que todos los estudiantes participen, manifiesten sus ideas, experiencias y cumpla con sus expectativas educativas. El centro de la educación es el alumno, guiado por su maestro. Solé (2011), afirma que la lectura constructiva alude a un proceso en el que el lector va armando mentalmente (construyendo) un modelo de texto, un significado o interpretación personal; esta actividad se puede lograr a lo largo de toda su vida, mientras lean buenos libros, más aguzadas serán sus destrezas. Por ese motivo los maestros deben enseñar estrategias pertinentes, actualizarse, estar comprometidos con su formación profesional en respuesta a los intereses y necesidades de sus educandos (Gavilán y Alario. 2010).

La aplicación de las estrategias Constructivistas de Inglés permite que los estudiantes desarrollen las cuatro habilidades: Escuchar, hablar, leer y escribir. Además, mejoraron su rendimiento y su capacidad comunicativa. La educación debe orientarse hacia el pleno desarrollo de la personalidad humana, hacia su dignidad y fortalecer el respeto por los derechos humanos. Según el informe de la UNESCO los contenidos tienen que fomentar el deseo permanente de aprender, el ansia y la alegría de conocer, así como la capacidad de comprender lo que se lee (Delors, 1996). El propósito fundamental del presente trabajo es demostrar la eficacia del empleo de la estrategia constructivista para mejorar la comprensión lectora en los tres niveles: Literal, inferencial y crítico-valorativo en el idioma inglés en los estudiantes de Educación Básica Alternativa, ya que la mayoría no lee, o, generalmente leen periódicos que no garantizan un nivel adecuado de lenguaje culto. Algunos diarios presentan mala redacción, pobreza gramatical y lenguaje 
soez característico de estos textos. Por eso, se asume un plan para involucrarlos en la lectura de textos literarios o no, pero que desarrolle en ellos su capacidad de retención, deducción-inducción, opinión y creatividad, ya que a partir del texto deben desarrollar sus habilidades lectoras.

\section{METODOLOGÍA}

Para el estudio se seleccionó una muestra de 32 estudiantes (15 mujeres y 17 hombres), hablantes nativos del español (14), quechua (16) y aymara (2); éstos últimos tienen como segunda lengua el castellano o español. Las edades fluctúan entre los 16 a 29 años. Los sujetos están agrupados al azar en dos secciones: "A" y "B". No se encontraron diferencias significativas entre sexo y el año escolar $\mathrm{x} 2=$ $2.42 \mathrm{p}=.08$; es decir, existe la misma proporción entre mujeres y varones de ambas secciones. El uso de estrategias Constructivistas utilizada en la aplicación de un Módulo para mejorar la comprensión lectora en inglés consiste en ir incorporando nuevos conocimientos a partir de los que ya tiene. En el ciclo Avanzado (secundaria) los estudiantes presentan dominios muy sencillos de gramática, ortografía y redacción, y muchas veces ellos no los consideran importantes, sin embargo, son fundamentales para ampliar su vocabulario en Castellano e Inglés, las comas, punto y coma, dos puntos y punto seguido; tienen un rol que los estudiantes deben ir identificándolos. Emplear estrategias de antes, durante y después de la lectura (Pinzás, 2012) y responder a preguntas literales, inferenciales y crítico valorativas, permiten asegurar que efectivamente los estudiantes comprendieron el texto.

Al inicio de cada clase se presentaron unas láminas, vinculadas con la lectura que se iba a trabajar. Observaron, describieron, respondieron preguntas, crearon nuevas situaciones, etc. Por ejemplo: ¿Qué observan? ¿Qué vínculos crees que existan entre los personajes? ¿De qué crees que están conversando? Se desarrolló un vocabulario en castellano y en inglés, se practicó con ellos la pronunciación.

La habilidad de escuchar significa comprender el sentido de lo percibido auditivamente (Marín, 2013). Los estudiantes identificaron oralmente en inglés las vocales, consonantes, palabras y frases; gracias a las prácticas continuas de reconocimiento. Pronunciaron términos, parafrasearon, La metodología participativa e interactiva ayudó a que las clases sean más interesantes para ellos y un reto constante para vencer sus temores, desafiar sus inseguridades y apostar por aprender constructivamente a partir de sus experiencias previas (Coll y otros, 2012).

Los estudiantes del grupo experimental realizaron diversos ejercicios de inspiración y espiración, por ejemplo, apagaron una vela encendida desde distancias gradualmente mayores. Soplaron una bolsa plástica hasta llenarla de aire. En una canaleta de carrizo se coloca una canica y dos estudiantes soplaron desde los extremos intentando empujar la bolita al campo contrario. Desarrollaron 
ejercicios labiales (masajes, extender y contraerlo, movimiento rotativo). Utilizaron un lápiz que fue sostenido con los labios para decir las palabras y textos breves. Todo esto permite al estudiante conocer y valorar su cuerpo y el esfuerzo por desarrollar actividades constructivistas que le van a permitir una auténtica destreza comunicativa (Desinano y Avendaño, 2016) venciendo su miedo e inseguridad, siendo tolerante con su persona y desarrollando su habilidad para hablar con seguridad. A diferencia del grupo de control que solo se le indica que hable o lea más fuerte, que no tenga temor de expresarse y que participe más en la clase.

La comprensión lectora se realizó teniendo en cuenta los tres niveles (MINEDU, 2015): Literal, inferencial y crítico valorativo. Los textos fueron breves, los estudiantes respondieron después de haber procesado y comprendido las preguntas. El análisis de imágenes de las lecturas les facilitó, conocer el significado de las palabras y aplicaron las estrategias: Antes, durante y después de la lectura (Solé, 2004). Desarrollaron las interrogantes literales, ¿Quién(es)? ¿Dónde? ¿Cuándo? ¿Qué? ¿Cómo? Las respuestas son breves, solo de palabras o frases textuales, es decir, con los mismos términos del texto. Ejemplo: Who is Jhon? A Teacher (¿Quién es Juan? Un profesor).

Contestar preguntas inferenciales, permite a los estudiantes interactuar con el texto, obteniendo una reflexión más profunda (Pinzás, 2012). Son interrogantes de un nivel más elevado fomentándoles el uso de sus propias palabras y mejora la redacción. Se emplea frecuentemente la expresión: ¿Por qué? Por Ejemplo: Why the students are working? (¿Por qué los estudiantes están trabajando?).

Las preguntas crítico-valorativas capacitan a los estudiantes para la reflexión, el sentido de opinión, piense y enjuicie el mensaje que ha recibido; para que no sea un simple repetidor de la información recibida (Cairney, 2011). Por ejemplo, ¿Qué hubieras hecho tú si...? ¿Qué opinas? ¿Qué le dirías a... de su actitud con sus padres? ¿Crees que actualmente se presentan esos casos? ¿Te parece bien que las familias sean desalojadas de sus viviendas porque viven cerca de una mina? Do you think that Robert is a nice friend? Why? (¿Piensas que Roberto es buen amigo? ¿Por qué?).

Los estudiantes lograron mejorar su habilidad para escribir oraciones simples en inglés, desarrollaron juego de palabras, dictado de términos, elaboraron trabajos individuales y en equipo; permitiéndoles redactar con cohesión, coherencia, mayor seguridad y creatividad para escribir; esto gracias a la aplicación de diversas estrategias. Como señala Solé (2011) la lectura es un reto para el estudiante, pero, especialmente para el docente para renovar y actualizar la manera de presentar las diversas lecturas a sus alumnos haciéndoles vivir la esencia de los personajes y la trama del texto, así como su capacidad de recrear las historias con la redacción de oraciones y párrafos. 
Teniendo en cuenta los aportes de Sánchez (2009) para fortalecer la enseñanza del idioma inglés se utilizaron diversos métodos como el gramática-traducción o tradicional, que pone mayor énfasis en la sintaxis de la lengua inglesa. El Método directo, establece un vínculo directo entre la palabra con la realidad a la que representa; permite la expresión oral y la memorización de vocabulario. Método audio-oral, prioriza el uso de la lengua hablada (audición y expresión oral) a partir de la repetición. El Método audiovisual, emplea los recursos visuales y auditivos para desarrollar la capacidad de escucha y de comprensión del lenguaje hablado a través de la interacción con sus compañeros. Aprendizaje basado en proyectos, busca la participación activa, la motivación y el trabajo en equipo.

El Marco Común Europeo otorga un especial énfasis al uso de estrategias pedagógicas que potencien el enfoque comunicativo a través de la oportuna planificación, implementación, seguimiento y evaluación, con la finalidad de que los estudiantes puedan expresarse adecuadamente (Férez, 2011). Los estudiantes adultos reconocen que la adquisición del inglés ofrece la ventaja de facilitarle muchas salidas profesionales (Arenas, 2011).

\section{Enfoque}

El trabajo es descriptivo explicativo, se trabajaron con dos variables. La variable dependiente: Mejorar la Comprensión Lectora en inglés.

La variable independiente: Módulo de estrategias Constructivistas.

\section{Participantes}

Intervinieron los estudiantes del primer año del ciclo avanzado, distribuidos al azar de la siguiente manera: El grupo experimental (sección B) constituido por 16 estudiantes de ambos sexos y al de control o tradicional (sección A), también formado por 16 alumnos de ambos sexos, cuyas edades fluctúan entre los 16 y 29 años.

\section{Instrumentos}

Se trabajaron los siguientes:

1. Texto: "A busy day in the office" del libro: "Estudios de Inglés" de Eugene J. Hall (Es um reactivo).

Ficha Técnica:

Propósito: Medir la habilidad general de comprensión lectora expresada en tres niveles: Literal, inferencial y crítico-valorativo.

Adaptación: está estandardizada a la realidade peruana. 
Administración: individual o colectivamente.

Usuarios: Sujetos de 12 a 40 años que cursen Educación Básica Alternativa o Regular (Secundaria)

Duración: 45 minutos, incluyendo el tiempo para dar instrucciones.

Corrección: Manual.

Puntuación: En el Nivel Literal seis preguntas, un punto cada una de ellas, nivel inferencial, cuatro preguntas, dos puntos cada ítem y el nivel crítico-valorativo tres preguntas cada uno de ellos dos puntos.

Descripción: La medición de la comprensión lectora mediante este texto se fundamenta en una definición de la comprensión lectora como una capacidad que se manifiesta a través de las subcapacidades: identificar el tema, los subtemas, la idea principal y la idea secundaria; reconocer e interpretar los hechos, inferir el autor, el contexto, etc.

\section{Validez y confiabilidad}

El concepto de validez implica que se deben tener intenciones claras y declaradas sobre dichas interpretaciones y consecuencias, de manera que estas puedan ser contrastadas con la evidencia disponible. A su vez, supone definir con precisión el o los constructos a evaluar (AERA, APA y NCME, 2014). La validez de un instrumento significa que debe medir la característica o variable por la que fue construido (Briones, 2004). Según el juicio de expertos la validez es de 0.85 . La confiabilidad se entiende como la consistencia de los resultados de una prueba a través de múltiples aplicaciones independientes (AERA, APA y NCME, 2014).

\section{Tabla 1}

Análisis de Confiabilidad de la Prueba "A busy day in the office"

\begin{tabular}{lccc}
\hline Ítems & Media & D.E. & ritc \\
\hline Literal: $1,2,3,4,5,6$ & 24.34 & 4.46 & 0.86 \\
Inferencial: 7.8 .9 .10 & 19.46 & 3.55 & 0.79 \\
Crítico-valorativo: $411,12,13$ & 18.73 & 3.48 & 0.77 \\
Alfa de Cronbach $=0.89$ & & & \\
\hline
\end{tabular}

Fuente: Análisis de datos

Fecha: mayo 2016

Los resultados del análisis generalizado de la confiabilidad de la Prueba: "A busy day in the office", incluye los niveles: Literal, inferencial y críticovalorativo, permiten apreciar que los ítem-test corregidas son superiores a 0.20 , lo que indica que los ítems son consistentes entre sí. El análisis de la confiabilidad 
por consistencia interna a través del coeficiente Alfa de Cronbach asciende a 0.89 , lo que permite concluir que la Prueba "A busy day in the office" presenta confiabilidad.

1. Análisis de imágenes.- El objetivo es que los estudiantes desarrollen las habilidades de escuchar, hablar y escribir, a través de las cuales se elaboraron preguntas que permitieron saber si reconocían objetos, situaciones, etc., haciendo las clases activas.

2. Fichas de lectura.- Este instrumento se empleó en el desarrollo de las técnicas para la enseñanza de la comprensión lectora. Cada ficha contiene tres rubros de preguntas que responden a los niveles:

3. Literal, inferencial y crítico-valorativo. Se aplicaron seis fichas al grupo experimental con una metodología constructivista - comunicativa siendo evaluadas en la escala vigesimal. Reconocieron la intencionalidad del texto.

4. Módulo de estratégias Construtivistas: Para elaborar este módulo se seleccionaron diversos textos de temas variados, que fueron escogidos con mucho cuidado dado su trascendencia en el trabajo con los estudiantes, del libro: "Estudios de Inglés" de Eugene J. Hall.

Las fichas de lectura, así como las pruebas de entrada y salida tienen la siguiente estructura: Nivel de lectura: Literal, inferencial y crítico-valorativo. La duración de la prueba fue de 45 minutos (Una hora pedagógica).

\section{RESULTADOS Y DISCUSIONES:}

El análisis de la eficacia del programa se realizó mediante las diferencias de las medias/medianas de las mediciones obtenidas antes y después de la aplicación del programa de intervención. Previamente se realizó una revisión a los descriptivos de la muestra y su el ajuste los datos a la normalidad mediante la prueba de Shapiro-Wilk. A partir de ello se decidió la utilización de un análisis paramétrico o no paramétrico acorde al caso.

En la Tabla 2 se observa que en el grupo control y en el grupo experimental existe un cambio en los puntajes de la promedios de cada dimensión antes y después de la intervención; las diferencias en el grupo control tienen un máximo de 1.77 puntos, mientras que en el grupo experimental, la diferencia máxima encontrada es de 3.05 en la nota final alcanzada. En general, los puntajes posteriores a la intervención en el grupo experimental son superiores a las del grupo control, a excepción de la dimensión crítico-valorativo. El puntaje de la nota final alcanzada presenta mayor diferencia entre ambos grupos. 
Tabla 2

Estadística descriptiva de las dimensiones a evaluar antes y después de la intervención de acuerdo a las condiciones experimentales

\begin{tabular}{lcccc}
\hline \multirow{2}{*}{ Dimensiones } & \multicolumn{3}{c}{ Pre-Test } & \multicolumn{3}{c}{ Post-Test } \\
\cline { 2 - 5 } & $\mathbf{M}$ & DE & M & DE \\
\hline Literal & 3.00 & 0.76 & 3.60 & 0.91 \\
Inferencia & 3.13 & 1.13 & 3.00 & 1.30 \\
Crítico-valorativo & 2.80 & 0.86 & 4.00 & 0.99 \\
Nota final alcanzada & 8.93 & 1.87 & 10.7 & 1.95 \\
\hline & & Grupo Experimental & \\
\hline Literal & 3.00 & 0.75 & 4.00 & 0.89 \\
Inferencia & 3.31 & 1.08 & 4.50 & 1.02 \\
Crítico-valorativo & 3.00 & 0.704 & 4.00 & 0.79 \\
Nota final alcanzada & 10.0 & 1.56 & 13.5 & 1.67 \\
\hline
\end{tabular}

Fuente: Registro de Evaluación de Notas Entrada y Salida.

Fecha: Mayo y octubre 2016

Posterior a la revisión descriptiva de los puntajes en cada dimensión antes y después de la intervención del programa en ambos grupos, se analiza de forma inferencial las diferencias.

\section{Tabla 3}

Diferencia y magnitud, antes y después de la intervención de las dimensiones a evaluar de acuerdo a las condiciones experimentales

\begin{tabular}{lccccccc}
\hline & \multicolumn{3}{c}{ Grupo Control } & \multicolumn{3}{c}{ Grupo Experimental } \\
\cline { 2 - 7 } Dimensiones & W & $\mathbf{p}$ & $\mathbf{d}$ & $\mathbf{W}$ & $\mathbf{p}$ & $\mathbf{d}$ \\
\hline Literal & 60.00 & .008 & 0.95 & 131.50 & $<.001$ & 1.71 \\
Inferencia & 31.50 & .041 & 0.63 & 120.00 & $<.001$ & 2.18 \\
Crítico-valorativo & 55.00 & .003 & 1.24 & 91.00 & $<.001$ & 1.58 \\
Nota final alcanzada & 120.00 & $<.001$ & 2.46 & 136.00 & $<.001$ & 3.09 \\
\hline
\end{tabular}

Fuente: Registro de Evaluación de Notas Entrada y Salida.

Fecha: Mayo y octubre 2016

En la tabla 3 se observa que las medidas antes y después de la intervención de las dimensiones literal, inferencia, crítico-valorativo y la nota final alcanzada presentan diferencias estadísticamente significativas tanto en el grupo control como en el experimental. Al evaluar la magnitud del efecto se evidencia que para todas las dimensiones mencionadas se tiene una magnitud grande tanto en el grupo control como en el experimental, a excepción de la dimensión inferencia donde el grupo experimental presenta una magnitud grande, mientras que el grupo control 
presenta una magnitud mediana. Además, en ambos grupos las dimensiones presentan diferencias estadísticamente significativas $(\mathrm{p}<0.05)$ entre sus puntajes pre-test y post-test.

\section{Tabla 4}

Diferencia y magnitud, antes de la intervención de las dimensiones a evaluar entre las condiciones experimentales

\begin{tabular}{lcccc}
\hline & \multicolumn{4}{c}{ Pre-Test } \\
\cline { 2 - 5 } Dimensiones & $\mathbf{t}_{\text {welch }}$ & $\mathbf{t}$ & $\mathbf{p}$ & d de Cohen \\
\hline Literal & 0.69 & & 0.494 & 0.25 \\
Inferencia & & 0.45 & 0.654 & 0.17 \\
Crítico-valorativo & & 1.82 & 0.079 & 0.65 \\
Nota final alcanzada & 1.42 & & 0.168 & 0.51 \\
\hline
\end{tabular}

Fuente: Registro de Evaluación de Notas Entrada y Salida.

Fecha: Mayo y octubre 2016

Se utilizó el estadístico t de Student para aquellas dimensiones que cumplían con los supuestos de normalidad e igualdad de varianzas; y se utilizó t de Welch para aquellos que infringían solo el supuesto de normalidad. Las medidas previas a la intervención indican que no existen diferencias estadísticamente significativas entre el grupo control y el grupo experimental a excepción de la dimensión críticovalorativo $(\mathrm{p}<0.05)$, la magnitud de esta diferencia es moderada $(\mathrm{d}=0.65)$.

\section{Tabla 5}

Diferencia y magnitud, después de la intervención de las dimensiones a evaluar entre las condiciones experimentales

\begin{tabular}{lcccc}
\hline \multirow{2}{*}{ Dimensiones } & \multicolumn{4}{c}{ Post-Test } \\
\cline { 2 - 5 } & 'Welch & $\mathbf{t}$ & $\mathbf{p}$ & d de Cohen \\
\hline Literal & 2.97 & & 0.006 & 1.07 \\
Inferencia & 2.58 & & 0.016 & 0.94 \\
Crítico-valorativo & 2.41 & & 0.023 & 0.87 \\
Nota final alcanzada & & 4.35 & $<.001$ & 1.56 \\
\hline
\end{tabular}

Fuente: Registro de Evaluación de Notas Entrada y Salida.

Fecha: Mayo y octubre 2016

Las medidas posteriores a la evaluación muestran diferencias estadísticamente significativas comparándolos de acuerdo a la condición experimental, siendo las medidas pertenecientes al grupo experimental las que mayor puntuación presentan en todas las dimensiones. La magnitud del efecto que apoyan las diferencias en las dimensiones literal, inferencia, critico-valorativo y nota final alcanzada $(\mathrm{d}=1.07$, $0.94,0.87,1.56$, respectivamente) es de tamaño grande. 


\section{Criterios de Clasificación}

Para el análisis de comparación de grupos independientes se reporta la d de Cohen el cual se interpreta de acuerdo a los siguientes criterios (Cohen, 1988): Trivial (d $<0.20)$, Pequeño $(0.20 \leq \mathrm{d}<0.50)$, Mediano $(0.50 \leq \mathrm{d}<0.80)$ y Grande $(\mathrm{d} \geq 0.80)$.

Después de haber aplicado el Módulo de estrategias Constructivistas, el promedio de la nota de la prueba de salida es superior. Los maestros tienen la responsabilidad de crear un ambiente de interés para enseñar la Comprensión Lectora (Reeve, 2010).

El texto empleado para medir la comprensión lectora a la entrada y salida del grupo de control y el experimental fue: "A busy day in the office". Los estudiantes identificaron las ideas con un poco de dificultad. A través de preguntas reconocieron el contenido y mensaje de la lectura. La sintaxis es importante pero no imprescindible para darnos cuenta que se ha comprendido un texto (Smith, 2008).

Las lecturas con imágenes permiten que los alumnos activen sus conocimientos previos e interactúen entre ellos comentando acerca de lo que perciben, se muestren activos con la lectura observando palabras, relacionándolas y sacando sus conclusiones (Díaz Barriga y Hernández 2010).

\section{DISCUSIÓN}

En los resultados se aprecia que la comprensión lectora en el grupo experimental es considerablemente superior que en el grupo de control. Es decir, sí hay correspondencia con los principios de las estrategias Constructivistas, el docente realiza diversos ejercicios con sus estudiantes antes de leer los textos y, a partir de ellos realiza diversas preguntas, actividades que le permitirán participar, expresar sus ideas, interactuar con sus compañeros, y, se tendrán en cuenta antes de iniciar la lectura. Concordando con los estudios de Ullari, (2017), la comprensión lectora es mayor en el grupo experimental cuando se realizan con éxito este tipo de ejercicios.

Se evidencia que facilitar a los estudiantes la habilidad de relacionar los elementos de una imagen con la información explícita de un texto, permite adquirir seguridad, ubicarse en el contenido, en el tema, con los personajes, el tiempo, los lugares, etc.; da lugar a una mejora de la comprensión lectora de los estudiantes coincidiendo con lo señalado por el MINEDU (2015) y Pinzás (2012) que, realizar estas prácticas constituye la base para seguir con un análisis más profundo, analítico y abstracto.

Un considerable desarrollo en la comprensión lectora se aprecia en el grupo experimental que logró una alta puntuación, incrementándose en 5.94, en el nivel 
inferencial, permitiéndonos concluir que las preguntas ¿Por qué crees...? ¿Cómo hubieras reaccionado si...? ¿Por qué crees que...? Entre otras, promueve en los estudiantes recapacitar en diversas situaciones, potenciando su habilidad en esta dimensión, coincidiendo con los hallazgos de Arenas (2011), quien señala que se debe fomentar en los estudiantes un nivel reflexivo de lectura, ya que permite ponerse en el lugar del otro, en diferentes contextos.

\section{CONCLUSIONES}

1. Los estudiantes del Grupo Experimental mejoraron significativamente su comprensión de lectura en el idioma inglés ya que respondieron a las interrogantes literales, ¿Quién(es)? ¿Dónde? ¿Cuándo? ¿Qué? ¿Cómo?, debido a la aplicación de un Módulo de estrategias Constructivistas.

2. Los estudiantes del Grupo Experimental desarrollaron notablemente su comprensión lectora en el idioma inglés al resolver preguntas inferenciales respondiendo a las interrogantes: ¿Por qué? ¿Por qué viven debajo de un puente? Do you think that Robert is a nice friend? Why? (¿Piensas que Roberto es buen amigo? ¿Por qué?, gracias a la aplicación de un Módulo de estrategias Constructivistas.

3. Los estudiantes del Grupo Experimental mejoraron significativamente su comprensión de lectura en el idioma inglés ya que respondieron a las interrogantes crítico-valorativas, respondieron a las siguientes preguntas: ¿Qué hubieras hecho tú si...? ¿Qué opinas? ¿Crees que actualmente se presentan esos casos? ¿Te parece bien que las familias sean desalojadas de sus viviendas porque viven cerca de una mina? Do you think that Robert is a nice friend? Why? (¿Piensas que Roberto es buen amigo? ¿Por qué?), debido a la aplicación de un Módulo de estrategias Constructivistas. 


\section{ANEXOS}

Anexo 01: Ficha de Lectura $\mathrm{N}^{\circ} 1$ :

\section{Lámina $\mathrm{N}^{\circ} 1$}

Trabajando en la oficina

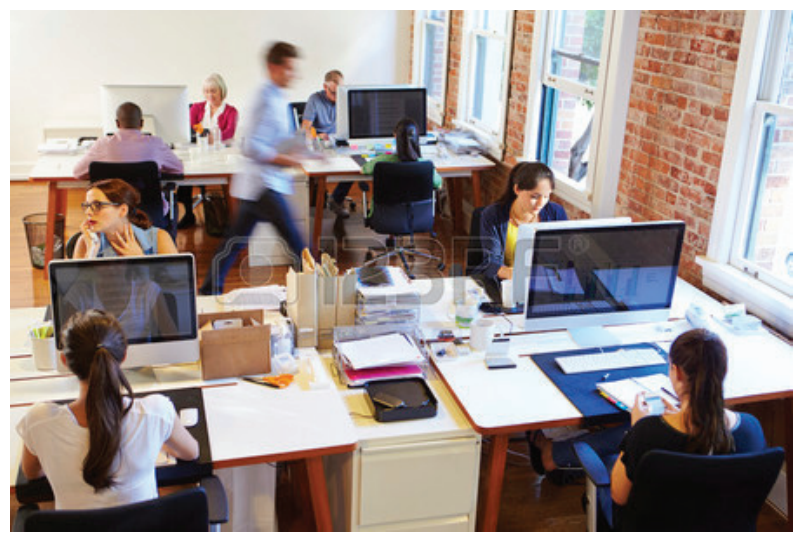

Fuente:http://www.elconfidencialdigital.com/dinero/Prohibido-videos-funcionarios-puestotrabajo_0_2532346753.html

Consultado el 20-03-16

Observa la lámina con mucho detenimiento. Se le formula las siguientes peguntas:

- ¿Qué aprecias en la imagen? ¿Quiénes son? ¿Dónde crees que están? ¿Qué hacen? ¿Cómo están vestidas?

- ¿Alguna vez has pensado ser secretaria? ¿Por qué? ¿A qué tipo de persona llamamos "formal" e "informal"? ¿Qué opinas de las personas formales e informales? ¿Por qué?

- ¿Qué objetos tienen las personas? ¿Crees que es un lugar ordenado o desordenado? ¿Por qué?

- ¿En qué empresas podemos encontrar este tipo de secretarias y oficinistas? ¿Crees que será agradable trabajar en un lugar así? ¿Por qué?

Después de escuchar sus respuestas, y de hacer un listado de palabras y expresiones primero en castellano y al costado en inglés, se procede a la pronunciación. Se pone énfasis en el volumen de voz, expresividad desarrollaron actividades de vocalización, inflexiones de voz, cambio de entonación y ritmo al leer (en inglés).

Los estudiantes leen dos veces en español y cuatro en inglés, la ejercitación bucal (abriendo y cerrando los labios) y facial: Exagerando la lectura, rostros alegres, tristes, pensativos, colocado un lápiz en la boca y leen los términos y expresiones. Luego, se procede a la lectura del texto: 
A busy day in the office

Anne Cooper is Mr. Johnson's secretary. She's typing a letter now. She's watching her work carefully because the letter is important. Mr. Johnson is very busy today. He is looking for someone to take his dictation. He has ten letters to answer. But everybody is busy. John is sending out the afternoon mail. Fred and Tom are filing correspondence. George is typing addresses on envelopes. He has two hundred envelopes on his desk. But Miss. Cooper is finishing the letter now. She's ready to take Mr. Johnson's dictation.

Después, responde las siguientes preguntas:

\section{LITERAL}

1. Who is Mr. Johnson's secretary?

2. What is she doing now?

3. How many letters does he have to answer?

4. What are Fred and Tom doing?

5. What's George doing?

6. How many envelopes are there on his desk?

\section{INFERENCIAL}

7. Why is Anne Cooper watching her work carefully?

8. What is Mr. Johnson's looking for?

9. What could Mr. Johnson do if Anne Cooper doesn't finish the letter?

10. What did you know about office work before reading this text?

\section{CRITICO - VALORATIVO}

11. Do you think the company should hire new clerks? (¿Crees que la empresa debe contratar nuevos empleados?)

12. Do you think that they always work this way in an office?

13. What kind of company do you think it is?

Finalmente, los estudiantes proceden a aumentar esta la historia en castellano, y luego con la ayuda de la profesora en inglés. Elaboraron dictado de palabras y frases. Redactaron de manera individual y en equipo oraciones en inglés con cohesión, coherencia y creatividad. Pegaron figuras relacionadas con el tema y en la parte inferior escribieron oraciones elaboradas por ellos. 
Anexo 02: Lista de Cotejo del Área de Inglés 2016

CEBA "Bartolomé Herrera"

UGEL 03

LISTA DE COTEJO 2016

Área: Inglés

Ciclo: Avanzado

Profesora: Maria Julia Alvarado Flores

Año y sección: $1^{0}$ "A"

\begin{tabular}{|c|c|c|c|c|c|c|c|c|c|c|c|c|c|c|c|}
\hline \multirow{3}{*}{$\begin{array}{l}\mathrm{N}^{\circ} \text { de } \\
\text { orden }\end{array}$} & \multirow{3}{*}{ Estudiantes } & \multicolumn{13}{|c|}{ Niveles de Comprensión Lectora } & \multirow{3}{*}{ Promedio } \\
\hline & & \multicolumn{6}{|c|}{ Literal (6puntos) } & \multicolumn{4}{|c|}{ Inferencial (8pts) } & \multicolumn{3}{|c|}{$\begin{array}{c}\text { Crítico- } \\
\text { Valorativo (6pts) }\end{array}$} & \\
\hline & & 1 & 1 & 1 & 1 & 1 & 1 & $2 \mathrm{pts}$ & $2 \mathrm{pts}$ & $2 \mathrm{pts}$ & 2 pts & $2 \mathrm{pts}$ & $2 \mathrm{pts}$ & $2 \mathrm{pts}$ & \\
\hline 01 & & & & & & & & & & & & & & & \\
\hline 02 & & & & & & & & & & & & & & & \\
\hline 03 & & & & & & & & & & & & & & & \\
\hline 04 & & & & & & & & & & & & & & & \\
\hline 05 & & & & & & & & & & & & & & & \\
\hline 06 & & & & & & & & & & & & & & & \\
\hline 07 & & & & & & & & & & & & & & & \\
\hline 08 & & & & & & & & & & & & & & & \\
\hline 09 & & & & & & & & & & & & & & & \\
\hline 10 & & & & & & & & & & & & & & & \\
\hline
\end{tabular}

Fuente: Aporte de la docente Maria Julia Alvarado Flores

marzo 2016 


\section{REFERENCIAS}

AERA, APA y NCME, (2014). Standards for Educational and Psychological Testing. Washington DC: American Educational Research Association.

Aguilar, J. (2015). "Programas educativos compensatorios en México. Problemas de equidad y de conocimiento", Perfiles Educativos (México), vol. XXXVII, núm. 147, pp. 183-200.

Akbari, M. (2013). Foreign Language Learninig. Recuperado el 16 de mayo de 2018, página web: http://www.irjabs.com/files_site/paperlist/r_1029_130815161601.pdfxdd

Arenas, J. (2011). "La relación entre las creencias y el incremento del filtro afectivo en el aprendizaje de inglés como lengua extranjera", Voces y Silencios: Revista Latinoamericana de Educación (Colombia), vol. 2, núm. 2, pp. 96-110.

Briones, G. (2004). La investigación social y educativa. Bogotá: Convenio Andrés Bello.

Cairney, T. (2011). Enseñanza de la comprensión lectora. Madrid: Morata Ediciones.

Cohen, J. (1988). Statistical Power Analysis for the Behavioral Sciences. null (2nd Ed., Vol. null). Lawrence Erlbaum Associates, Publishers

Coll, C. y otros. (2012). El constructivismo en el aula. Barcelona: GRAÓ.

Crystal, D. (2003). English as a global language. Recuperado el 12 de abril de 2017, Cambridge University Press, página web de la Library of Congress classification outline: http://catdir.loc.gov/catdir/samples/cam041/2003282119.pdf

Declaración Mundial sobre Educación para Todos (1994). Satisfacción de las necesidades básicas de aprendizaje. (2da. Ed.). New York: UNESCO.

Delors, J. (1996). La Educación encierra un tesoro. Madrid: Santillana.

Delval, J. (2000). Aprender en la vida y en la escuela. Madrid: Ediciones Morata.

Desinano, N. y Avendaño, F. (2016). Didáctica de las ciencias del Lenguaje, enseñar ciencias del lenguaje. Rosario: Homo Sapiens Ediciones.

Díaz Barriga, F. y Hernández, G. (2010). Estrategias docentes para un aprendizaje significativo, una interpretación constructivista. (3ra. ed.). México: McGraw-Hill.

Férez, P. (2011). "La competencia estratégica en la enseñanza de la L2: El marco común europeo de referencia para las lenguas y new english file advanced", Cartaphilus (España), núm. 9, pp. 135-140.

Frisancho, S. (2016). Ensayos Constructivistas. (1ra. ed.). Lima: Fondo Editorial PUCP.

Ganem, P. y Ragasol, M. (2016). Piaget y Vygotski en el aula. El constructivismo como alternativa de trabajo docente. (1ra. ed.). México: Limusa.

García, A. (2008). Metodología de la enseñanza del inglés. Recuperado el 17 de junio de 2018, OTEC Instituto Anglo-Saxon. N.Ch.2728, página web: http:// institutoanglosaxon.blogspot.com/2008/11/metodologa-de-la-enseanza-del-inglspor.html 
Gavilán, P. y Alario, R. (2010). Aprendizaje cooperativo una metodología con futuro. Principios y aplicaciones. Madrid: CCS.

Gonzales, M. y otros (2016). Enseñar a leer y escribir en la educación superior. México: Universidad Autónoma de Tamaulipas.

Hall, E. (1965). Estudios de Inglés Curso Intermedio-Avanzado. New York: SIMON AND SCHUSTER.

Idrogo, G. y Huamán, A. (2018). Proyecto Institucional de Lectura PIL. Propuestas para su diseño y aplicación. Lima: Editorial San Marcos.

Marín, M. (2013). Lingüística y Enseñanza de la Lengua. Buenos Aires: Editorial Educación Aique.

Mendes, V. (2014). "¿Qué principio de justicia para la educación básica?", Cuadernos de Pesquisa (Brasil), vol. 44, núm. 154, pp. 1094-1109.

MINEDU. (2009). Ministerio de Educación del Perú. Diseño curricular básico nacional de Educación Básica Alternativa. Lima: Gobierno del Perú.

MINEDU. (2015). Ministerio de Educación del Perú. Rutas de Aprendizaje ¿Qué y cómo aprenden nuestros estudiantes? VI Ciclo Área Curricular de Comunicación, Fascículo 1. Lima: Gobierno del Perú.

Neyra, L. y Flores, J. (2011). Comprensión y producción textual. Lima: Editorial San Marcos.

Objetivos de Dakar (2012). Acercándonos a la situación de la educación en Centroamérica. Recuperado el 15 de junio de 2018: http:/www2.congreso.gob.pe/sicr/cendocbib/ con4_uibd.nsf/5C9142C44FA6CB6305257D7200519118/\$FILE/informe_objetivos de_dakar_situacion_de_la_educacion_en_centroamerica.pdf

Peirano, L. (2010). La importancia de hablar inglés. Recuperado el 28 de mayo de 2018, página PUCP: https://www.youtube.com/watch?v=K-pjJr97hDs

Pinzás, J. (2012). Leer pensando introducción a la visión contemporánea de la lectura. (3ra ed.). Lima: Fondo editorial Pontificia Universidad Católica del Perú.

Reeve, J. (2010). Motivación y emoción. (5ta ed.). México: McGraw Hill.

Ricoy, M. y Alvarez, S.(2016). La enseñanza del inglés en la educación básica de personas jóvenes y adultas. Recuperado el 08 de agosto de 2017, página web: http:// www.scielo.org.mx/scielo.php?script=sci_arttext\&pid=S1405-66662016000200385

Sánchez, M. (2009). Historia de la metodología de enseñanza de lenguas extranjeras. Recuperado el 08 de agosto 2017 file://C:/Users/MARIA\%20ALVAVARADO/ Downloads/Dialnet-HistoriaDeLaMetodologiaDeEnsenanzaDeLenguasExtra nj-2983568.pdf

Sanz, J.; Sanz, F.; Jiménez, R. y Lancho, J. (2009). Educación de personas adultas en el marco del aprendizaje a lo largo de la vida, Madrid: UNED.

Smith, F. (2008). Comprensión de la lectura: Análisis psicolingüístico de la lectura y su aprendizaje. México: Trillas. 
Solé, I. (2004). Estrategias de Lectura. (15va ed.). Barcelona: Grao ICE.

Solé, I. (2011). Comprensión lectora y aprendizaje significativo. Barcelona: Editorial de la Universidad de Barcelona.

Suárez, R. (2011). La Educación, Estrategias de enseñanza-aprendizaje, Teorías educativas. México: Trillas.

Ullari, M. (2017). Influencia de los estilos de aprendizaje en el rendimiento académico del idioma inglés de los estudiantes del centro de idiomas de la Facultad de Ciencias de la Educación, humanas y tecnológica de la Universidad Nacional de Chimborazo 2015-2016. Lima: UNMSM.

UNESCO (1948). Declaración Universal de los Derechos Humanos.Recuperado el 14 de junio 2018, página web: http://www.derechoshumanos.net/normativa/normas/1948DeclaracionUniversal.htm?gclid=CjwKEAiA48fDBRDJ24_imejhwUkSJAAr0M5k kJ46W4PTG5ItvIVjpLaaSZdIDicLiwYgTB004GKmQBoCq̄J7w_wcB 
